\title{
CO-RELATION AMONG LEISURE-TIME PHYSICAL ACTIVITIES AND HEALTH-RELATED QUALITY OF LIFE IN PHYSICAL THERAPY STUDENTS
}

\begin{abstract}
INTRODUCTION

Physical inactivity has become a global burden that leads to adverse health conditions in particular obesity, hypertension, and diabetes. Moreover, the modern generation tends to have a sedentary lifestyle because of the burden and increasing usage of technologies including cell phones, laptops, or gaming gadgets. Therefore, students lack Physical Activity (PA) even in their leisuretime due to fatigue and a decrease in Quality of Life (QoL)
\end{abstract}

\section{METHODOLOGY}

A cross-sectional survey was conducted using convenience sampling technique among physical therapy students. Godin-Leisure Time Questionnaire and World Health Organization Quality of Life Instrument (WHO HRQOLBREF) questionnaire was used to collect data and sent to participants via email or WhatsApp groups.

\section{RESULTS}

A total number of 384 physical therapy students participated in the study with 104 (27\%) males and 280 females $(73 \%)$ respectively. The survey showed $74 \%$ of individual's active followed by $19.2 \%$ moderately active while only $1.4 \%$ sedentary. An $81.1 \pm 11.4$ average was attained on the HRQoL that suggested individuals have a good quality of life. Besides, no significant association $(p=0.156)$ between leisure-time activity and health-related quality of life (HRQoL) of physical therapy students was found.

\section{CONCLUSION}

It was concluded that the majority of students were active in performing moderate PA followed by good QoL. Moreover, no significant association between leisure-time activity and HRQoL of physical therapy students was found therefore, large-scale multicenter trials should be conducted to evaluate the factors related to PA and QoL in young individuals considering standardized assessments to have reliable outcomes.

\section{KEYWORDS}

Physical activity, Exercise, Leisure-Time, Physical Activity, Quality of Life, Activities of Daily Living.
Mehwish Taskeen

Physiotherapist

Freelancer

Roshanmasi1234@gmail.com
[Taskeen M. Co-Relation Among Leisure-Time Physical Activities And Health-Related Quality Of Life In Physical Therapy Students.Pak.J .rehabil.2021;10(1):26-33] 10.36283/pjr.zu.10.1/005 


\section{INTRODUCTION}

Physical activity (PA) is determined as the movement that is produced by the body requiring energy for the activities for leisure time, traveling or occupational performances $^{1,2}$. There are several ways where an individual can be active that includes walking both on slow or fast speed, cycling, high and slow impact activities, sports, and different recreational activities that can be done for health as well as enjoyment purpose by children, adult, and geriatric population ${ }^{2}$. Along with physical activity which is a substantial component of life that significantly increases vital consumption, there are other factors that also have a positive correlation with vital consumption. These factors include encouragement of population towards fitness and provision of awareness to the public towards the actual definition and implementation of health and wellness ${ }^{3}$.

As far as relationship between leisure activities and physical health on the grounds of well-being is concerned, there is a study conducted by Paggie M.E. et al in 2016, in which database of 259 adults ranging from 18 to 81 years of age who had completed different questionnaires were taken and analyzed on the basis of mediation and linear regression models. Eventually, the results revealed a stronger relationship between leisure activities and physical health among individuals with compromised well being ${ }^{4}$.

Moreover, physical activities may also play a crucial role in the overall selfreported well-being of an individual. Hence, A meta-analysis conducted by Granger E. in 2017 among adolescents attempted to evaluate whether physical activity only improves health outcomes objectively or do they have an additional impact upon an individual's self-reported well-being. Eventually, nine out of eleven studies supported and proved that a significant positive relationship between physical activities and self-reported well beings of individuals was present. This meta-analysis also supported sub optimal levels of physical activities to be also effective in both state of objective wellbeing and self-perceptional well-being. Despite, several challenging health-values that have been associated with leisuretime such as diabetes mellitus, stroke, hypertension or coronary heart disease, etc., routine physical activity is considered extremely important for not only managing the disease as well as preventing multiple medical conditions ${ }^{5}$. In fact, patients with chronic kidney disease suffer from muscle atrophy that progresses with the combined effects of uraemia and physical inactivity. Upon these types of chronic diseases, Physical activities (PA) plays a vital role to negate the symptomatic progression of CKD. Moreover, around 1 in 3 women and 1 in 4 men worldwide do not engage themselves in enough physical activity to stay healthy ${ }^{1,6}$.

As reported by World Health Organization (WHO), more than a quarter of the global population is observed to be insufficiently active that may lead individuals towards acquiring noncommunicable diseases such as diabetes, stroke, heart diseases and cancer. In children, physical inactivity or sedentary lifestyle can promote excessive adiposity (weight gain) decreased cardiac health, compromised social behavior and sleep distortions ${ }^{2}$. Furthermore, physical inactivity is labeled as the fourth leading cause of mortality, accounting for $5.5 \%$ of deaths worldwide2.

Moreover, recent researches revealed that regular physical activities may be beneficial to eliminate the risk factors of these diseases therefore, it has been recommended that physical activities should be carried out regularly to avoid any health-related consequences and improve quality of life ${ }^{5}$. Furthermore, these activities may also be beneficial to eliminate negative features thereby enhancing physical, social, and cognitive 
components of health dimension ${ }^{7}$. Several researchers concluded that in students, teachers, or doctors, leisure-time activities are found to be less due to busy lifestyle and increase workload therefore it is important to provide them awareness on the positive impact of physical activity $^{8}$.

Moreover, Health-related quality of life (HRQOL) is considered as an important multidisciplinary concern for evaluating preventive or therapeutic health plans in research settings ${ }^{9}$.Several studies have concluded that daily activities have a positive impact on the excellence of life using HRQoL as a screening tool. Further, the connection between bodily exercises with HRQoL is found to be significant ${ }^{10}$. As observed in medical students that HRQoL is affected due to decreased PA that may lead to psychosomatic disorders ${ }^{11}$. Moreover, it has been discovered that physical activities and quality of life have been affiliated in subsidiary and are possibly interceded by factors like bodily relations, self-respect workout, and selfjudgment that are designed to encourage standard physical actions and surge selfconfidence that is compelling to progress in life further ${ }^{5}$. Further, recent researchers concluded that there is still scarce material on the connection amid particularly bodily actions and healthcare of daily living in particular individuals. It was also suggested that future researches should assess the connection among physical activities in certain fields such as areas of job, institutions, offices, home, and other aspects of healthcare professional's life ${ }^{12}$. The target population was evaluated based on the level of activity and value of life well-being and health facilities for working physiotherapists in clinical practice. Furthermore, it was thought that practitioners should report health-related quality of life and be physically energetic in their field. Such a discovery lead to the assumption that common physical activity musculoskeletal diseases among physiotherapists, perhaps, future reviews should focus on improving and assessing role-specific conditioning procedures ${ }^{13}$.Therefore, future studies should be based on these variables to evaluate the factors related to PA and QoL.

As PA is also recognized as a modifiable risk factor of behavior associated with the QoL and health, therefore, physical exercise should be promoted in individuals of all age group to have a may have an advantageous impact on reducing stress, depression, anxiety, or depression and for the healthy wellbeing ${ }^{14}$. Although, physical fitness levels are linked with the regularity of physical activity recommendations and lead to immense benefits when practice appropriately, therefore, in order to determine the association of leisure-time PA with HRQoL in physical therapy students ${ }^{15}$.

During the COVID-19 pandemic the world has seen a severe decline in physical inactivity as well as decreased quality of life of most individuals. For instance a study was conducted by Slimani M. et al during COVID lockdown among 216 individuals living in Tunisia. The study compared quality of life (by WHO Quality of Life Instrument-Short Form WHOQOLBREF) of three groups on the basis of activity levels including inactive group, minimally active group and those who performed health enhancing physical activities. The results revealed a positive correlation between physical activity levels and quality of life ${ }^{17}$.

\section{METHODOLOGY}

Study Design: A cross-sectional survey was conducted among physical therapy students.

Study Setting: The data was collected from academic institutes where the physical therapy students were enrolled in rehabilitation department in Karachi 
Duration of Study: 6 to 8 months.

Sampling Technique: 384 students were sampled through a non-probability convenience Sampling Technique

Inclusion criteria:

- Both male and female physical therapy students were included who were currently enrolled Physical Therapy degree program at Karachi rehabilitation academic institutes.

\section{Exclusion Criteria:}

- Students enrolled outside Karachi

- Students enrolled only in deficiency program

- Student registered in any regular sports activity such as football or cricket team.

\section{Data Collection:}

\section{Data Collection Tools:}

Data was collected through the World Health Organization Quality of Life Instrument (WHO HRQOL-BREF) that is an abbreviated version of WHQoL-100 and Godin Leisure-Time Questionnaire.

- WHOQOL-BREF was a commonly used questionnaire in quality of life that consists of four domains (psychological health, environmental health, physical health, and social relationships) ${ }^{16}$. Higher domain scores indicate higher quality of life. The domain score was computed by the mean score of all questions in each domain. Mean scores were then duplicated by 4 to make domain scores comparable to the scores utilized in the scale.

\section{- $\quad$ Godin Leisure-Time Questionnaire} was designed to measure the physical activity comprised of a 4-item scale which evaluates episodes, intensity, and duration of activity over a typical 7-day time-frame. The instrument had a good test-retest reliability ranging from 0.74 to 0.80 respectively.

\section{Data Collection Procedure:}

Due to the ongoing COVID-19 pandemic, data was collected through Google Docs consisted of compiled questionnaires based on mentioned tools that were sent to participants via email and social media platforms. Moreover, all participants were provided with informed consent to had detailed information about the study. The responses of participants on WHOQOL and Godin Leisure-Time questionnaires were evaluated to determine the association of Leisure-Time Physical Therapy with HRQoL.

Ethical Considerations: The researcher provided complete information about the study to every participant before the start of the study. The information procured from the participants was kept as a confidential information. Every participant had full right of withdrawing from the study at any time.

Data Analysis: Data were being entered and analyzed using SPSS (Statistical Package for Social Sciences) version 20. The demographic characteristics of the participants were being represented through frequency, mean and standard deviation whereas the association was being assessed through the Chi-Square Test of Association while $p<0.05$ was being considered significant.

\section{RESULTS}

A total number of 384 physical therapy students participated in the study with the highest age range reported in 20-25 years $(90.4 \%)$ with a female majority of 42 (11\%) and 342 (83.6\%) males and females respectively. Furthermore, out of 384 students, only 53 students were married. The demographic detail of participants is described in Table-1. 


\begin{tabular}{|c|c|}
\hline \multicolumn{2}{|c|}{$\begin{array}{l}\text { Table-1 Demographic Details of } \\
\text { Participants }\end{array}$} \\
\hline Variables & $N(\%)$ \\
\hline \multicolumn{2}{|l|}{ Age } \\
\hline $18-22$ years & 346 (90.4\%) \\
\hline $23-25$ years & $38(9.6 \%)$ \\
\hline \multicolumn{2}{|l|}{ Gender } \\
\hline Male & $104(27 \%)$ \\
\hline Female & $280(73 \%)$ \\
\hline \multicolumn{2}{|l|}{ Marital Status } \\
\hline Single & 331 (86.3\%) \\
\hline Married & $53(8.2 \%)$ \\
\hline
\end{tabular}

Table-2 Godin-Leisure Time Questionnaire

\begin{tabular}{lccc}
\hline & \multicolumn{3}{c}{ Interpretation } \\
& $\begin{array}{c}\text { Sedentary } \\
\text { (<14 Units) }\end{array}$ & $\begin{array}{c}\text { Moderately } \\
\text { Active } \\
(\mathbf{1 4 - 2 3} \\
\text { Units) }\end{array}$ & $\begin{array}{c}\text { Active } \\
(\mathbf{2 4} \text { or } \\
\text { More } \\
\text { Units) }\end{array}$ \\
$\begin{array}{l}\text { Weekly Godin } \\
\text { Leisure Time } \\
\begin{array}{l}\text { Questionnair } \\
\text { e Scores }\end{array}\end{array}$ & $1(1.4 \%)$ & $14(19.2 \%)$ & $54(74 \%)$ \\
$N=$ Frequency & & & \\
\hline
\end{tabular}

The screenings on Godin-Leisure Time Questionnaire revealed that $74 \%$ of individuals are active, followed by $19.2 \%$ moderately active while only 1.4 are sedentary. Moreover, 81.1 \pm 11.4 average was attained on the WHQoL that suggested individuals has a good quality of life i.e. 34 (46.6\%) reported good rating of the quality of life, 16 (21.9\%) neither good or bad, 15 (20.5\%), and only 4 (5.5\%) marked poor on the questionnaire. Furthermore, 36 (49.3\%) were found to be satisfied with their health, 15 (20.5\%) neutral, 9 (12.3\%) dissatisfied and 7 $(9.6 \%)$ very satisfied. The detail of participants is depicted in Table-3, 4 and 5.

The Chi-square test of association revealed no significant association between leisure-time activity and healthrelated quality of life of physical therapy students $(p=0.156)$ as shown in Table-3. Moreover, the majority of students were found to be physically active and have a better quality of life.

Table-3 Chi-Square Test of Association

\begin{tabular}{l|cc|} 
& Value & Sig. \\
Pearson Chi-Square & 12.6 & $* 0.941$ \\
Likelihood Ratio & 29.329 & 0.999 \\
\hline \multicolumn{3}{l|}{$p>0.05$ indicates insignificant association }
\end{tabular}

\section{DISCUSSION}

The findings of this study revealed $74 \%$ of individuals were active, followed by $19.2 \%$ moderately active while only $1.4 \%$ were sedentary. Moreover, an $81.1 \pm 11.4$ average was attained on the WHOQOLBREF that suggested individuals have a good quality of life. Besides, no significant association $(p=0.156)$ between leisuretime activity and health-related quality of life of physical therapy students was found. The key finding of our investigation was to determine a dose-response relationship between leisure-physical activity and QoL in physiotherapy students. Besides, to the best of the author's knowledge, no previous work has explicitly designed to determine the association between the aforementioned variables.

A study conducted by Peleiaset $\mathrm{al}^{17}$ showed a definite dose-response relationship between leisure-time physical activity with QoL dimensions in male and female students. The study suggested that amount of energy consumed during activities in daily life has not varied significantly. Moreover, METs spent on the average week were not estimated. However, our study evaluated physical activity duration on an average week with a standardized questionnaire but the cross-sectional nature of the study and self-reported measures may 
serve as a limitation. A large-scale multitrial was conducted to compare the effects of leisure-time and physical activity of community on blood pressure showed a high prevalence of hypertension among women who tended to be inactive during leisure time ${ }^{18}$.

Despite this, many studies that demonstrated an effect of consistency of diet on health-related quality of life in children found that healthier dietary habits are significantly correlated with higher nutritional status contributing to significant effects on physical functioning and psychosocial quality of life. Although, the association between the quality of life or dietary behaviour with health-related dimensions of quality of life is yet to be explored thoroughly ${ }^{16,19,20}$. On the other hand, Joseph et $\mathrm{al}^{21}$ investigated the mediating effects of exercise and physical self-esteem on the relationship between PA and QOL in a young adult population. This disparity in results are probably related to shifts in individual values that arise during life. Furthermore, several factors such as social pressure or financial pressure may also be a contributor to low quality of life. Therefore, younger individuals need to pay more attention to these aspects to enhance their quality of life ${ }^{22-24}$. Besides, students are also overburdened due to their on-going education and daily routine activities may hinder to perform physical activities even in leisure-time therefore to maintain a healthy lifestyle, physical activity should be promoted for a better quality of life ${ }^{25-}$ 27 . This study is the first to be conducted exploring student's physical activity levels in leisure time and quality of life compared to the study done in Tunisia ${ }^{17}$ that compared similar variables i.e. quality of life and physical activity levels among adults who were non-specifically students with mean age 28.2 years during the COVID-19 lockdown. Moreover, the concept contributed significant information towards the stakeholders to rule out the factors associated with physical activity impact on the quality of life in young individuals.

Since several limitations occurred during this study that included limited sample size due to the on-going COVID-19 pandemic so results cannot be generalized. Furthermore, our samples contained a significant majority of female students that could have elicited a gender biased results. Moreover, the selfreported measure may also lead to outcome bias. However, further studies are required on a national level to evaluate the factors related to physical activity and quality of life in young individuals of Pakistan. Furthermore, our study did not grouped individuals according to certain physical activity levels as aforementioned studies did. Moreover, a similar study that was done by Maugeri $G$ et al (2020) in Italy during COVID-19 lockdown compared physical activity levels with quality of life but with more detailed demographic data that included each individual's height, weight and $\mathrm{BMI}$ and grouped them on the basis of underweight and overweight ${ }^{29}$. Therefore, a future study similar to ours among students that could also include more detailed demographic data specially $\mathrm{BMI}$ and dietary intake can further evaluate more accurate relationship between physical activity and quality of life of participants.

\section{CONCLUSION}

It was concluded that the majority of students were active in performing moderately physical activities followed by the good quality of life. Moreover, no significant association between leisuretime activity and health-related quality of life of physiotherapy students was found therefore, large-scale multicentre trials should be conducted to evaluate the factors related to physical activity and quality of life in young individuals 
considering standardized assessments to have reliable outcomes.

\section{REFERENCES}

[1] Physical Activity: WHO; 2015 [cited 2016 26.02.2016]. Available at: http://www.who.int/mediacentre/fac tsheets/fs385/en/.

[2] Corbin CB, Pangrazi RP, Franks BD. Definitions: Health, fitness, and physicalactivity. President\&\#39;s Council on Physical Fitness and Sports Research Digest. 2000 Mar.

[3] Paggi ME, Jopp D, Hertzog C. The importance of leisure activities in the relationshipbetween physical health and well-being in a life span sample. Gerontology. 2016;62(4):450-8

[4] Granger E, Di Nardo F, Harrison A, Patterson L, Holmes R, Verma A. A systematicreview of the relationship of physical activity and health status in adolescents. European journal of public health. 2017 May 1;27(suppl_2):100-6.

[5] Zelle DM, Klaassen G, Van Adrichem E, Bakker SJ, Corpeleijn E, Navis G. Physicalinactivity: a risk factor and target for intervention in renal care. Nature ReviewsNephrology. 2017 Mar;13(3):152.

[6] Warburton DE, Bredin SS. Reflections on physical activity and health: what should we recommend?. Canadian Journal of Cardiology. 2016 Apr 1;32(4):495-504.

[7] Pangrazi P. R, Beighle A. Dynamic Physical Education for Elementary School Children.19th ed. 2019.

[8] Chang HC, Liang J, Hsu HC, Lin SK, Chang TH, Liu SH. Regular exercise and the trajectory of health-related quality of life among Taiwanese adults: a cohort analysis 2006-2014. BMC public health. 2019 Dec;19 (1):1-2.

[9] Yasunaga A, Shibata A, Ishii K, Inoue S, Sugiyama T, Owen N, Oka K. Replacing sedentary time with physical activity: effects on health-related quality of life in older Japanese adults. Health and quality of life outcomes. 2018 Dec;16(1):1-5.

[10] Oztasan N, Ozyrek P, Kilic I. Factors associated with health-related quality of life among university students in Turkey. Materia socio-medica. 2016 Jun;28(3):210.

[11] Pedišić Ž, Rakovac M, Titze S, Jurakić $D, \quad$ Oja P. Domain-specific physicalactivity and health-related quality of life in university students. European journal of sport science. 2014 Jul 4;14(5):492-9.

[12] McPhail SM, Waite MC. Physical activity and health-related quality of lifeamong physiotherapists: a cross sectional survey in an Australian hospital and health service. Journal of Occupational Medicine and Toxicology. 2014 Dec 1;9(1):1.

[13] Lok N, Lok S, Canbaz M. The effect of physical activity on depressivesymptoms and quality of life among elderly nursing home residents: Randomized controlled trial. Archives of gerontology and geriatrics. 2017 May 1;70:92-8.

[14] Tian $Y$, Jiang $C$, Wang $M$, Cai R, Zhang $Y$, He Z, Wang $H$, Wu D, Wang F, Liu X, $\mathrm{He} Z$ Z. BMI, leisure-time physical activity, and physical fitness in adults inChina: results from a series of national surveys, 2000-14. The Lancet Diabetes \&amp; Endocrinology. 2016 Jun 1;4(6):487-97.

[15] Wu XY, Zhuang LH, Li W, Guo HW, Zhang JH, Zhao YK, Hu JW, Gao QQ, Luo S, Ohinmaa A, Veugelers PJ. The influence of diet quality and dietary behavior on health-related quality of life in the general population of children and adolescents: a systematic review and meta-analysis. Quality of Life Research.2019 Aug 1:1-27.

[16] Slimani $M$, Paravlic A, Mbarek F, Bragazzi NL, Tod D. The relationship between physical activity and quality of life during the confinement induced by COVID-19 outbreak: a pilot study in 
Tunisia. Frontiers in psychology. 2020;11.

[17] Peleias $M$, Tempski $P$, Paro HB, Perotta B, Mayer FB, Enns SC, Gannam S, Pereira MA, Silveira PS, Santos IS, Carvalho CR. Leisure time physical activity and quality of life in medical students: results from a multicentre study. BMJ open sport \&amp; exercise medicine. 2017 Jun 1;3(1):e000213.

[18] Treff C, Benseñor IM, Lotufo PA. Leisure-time and commuting physical activity andhigh blood pressure: the Brazilian Longitudinal Study of Adult Health (ELSA-Brasil). Journal of human hypertension. 2017 Apr;31(4):278-83.

[19] Xiao $Y$, Wang $H$, Zhang $T$, Ren $X$. Psychosocial predictors of physical activity and health-related quality of life among Shanghai working adults. Health and quality of life outcomes. 2019 Dec;17(1):1-9.

[20] Zhang T, Xiang P, Gu X, Rose $M$. College Students\&\#39; Physical Activity and Health- Related Quality of Life: An Achievement Goal Perspective. Research Quarterly for Exercise and Sport. 2016 Apr 2;87(2):182-90.

[21] Joseph RP, Royse KE, Benitez TJ, Pekmezi DW. Physical activity and quality of life among university students: exploring self-efficacy, selfesteem, and affect as potential mediators. Quality of life research. 2014 Mar 1;23(2):659-67.

[22] Alzahrani $H$, Cheng SW, Shirley $D$, Mackey M, Stamatakis E. Physical activity and health-related quality of life in people with back pain: a population-based pooled study of 27,273 adults. Journal of Physical Activity and Health. 2020 Feb 1;17(2):177-88.

[23] Bae WS, Lee HO, Shin JW, Lee KC. The effect of middle and lower trapeziusstrength exercises and levator scapulae and upper trapezius stretching exercises in upper crossed syndrome. Journal of physical therapy science. 2016;28(5):1636-9.

[24] Lawan A, Awotidebe AW, Oyeyemi AL, Rufa AA, Oyeyemi AY. Relationship between physical activity and health related quality of life among pregnant women. African journal of reproductive health. 2018;22(3):80-9.

[25] Nowak PF, Bożek A, Blukacz M. Physical Activity, Sedentary Behavior, and Quality of Life among University Students. BioMed Research International. 2019 Dec 18;2019.

[26] Ou HT, Su CT, Luh WM, Lin CY. Knowing is half the battle: the association between leisure-time physical activity and quality of life among four groups with different selfperceived health status in Taiwan. Applied Research in Quality of Life. 2017 Dec 1;12(4):799-812.

[27] Subramaniam $M$, Zhang $Y$, Lau JH, Vaingankar JA, Abdin E, Chong SA, Lee ES. Patterns of physical activity and health-related quality of life amongst patients with multimorbidity in a multi-ethnic Asian population. BMC Public Health. 2019 Dec 1;19(1):1612.

[28] Maugeri G, Castrogiovanni P, Battaglia G, Pippi R, D'Agata V, Palma A, Di Rosa $M$, Musumeci $G$. The impact of physical activity on psychological health during Covid-19 pandemic in Italy. Heliyon. 2020 Jun 1;6(6):e04315. 